\title{
Estimación de la Talla a Partir de la Medida de la Tibia en Población Colombiana
}

\author{
Estimation of Height from Measurements of the Tibia in Colombian Population \\ *Julio Cesar Mantilla Hernández; **Nelson Cárdenas Durán; ***Jesús Maria Jácome Bohórquez
}

MANTILLA, H. J. C.; CÁRDENAS, D. N. \& JÁCOME, B. J. M. Estimación de la talla a partir de la medida de la tibia en población Colombiana. Int. J. Morphol., 27(2):305-309, 2009.

RESUMEN: La desaparición forzada es un fenómeno común en Colombia, diariamente aparecen restos humanos en fosas comunes, el estado tiene la responsabilidad de identificar tales restos, el proceso inicial de identificación debe determinar: género, raza, edad y talla. Nuestro objetivo es establecer la correlación existente entre la longitud de la tibia y la talla de un individuo con el fin de generar una ecuación que permita predecir la talla de un individuo a partir de los restos óseos de su tibia. Se evaluaron 168 cadáveres a quienes se les practicó la autopsia medico-legal, con edades comprendidas entre los 18 y 50 años. Se realizó la medición de la talla del sujeto y de la longitud máxima de su tibia luego de su disección, y se estimó la correlación existente entre estas dos variables a partir del coeficiente de correlación de Pearson y se aplica un modelo de regresión lineal para generar una ecuación que permita predecir la talla de un individuo a partir de la longitud de su tibia usando el paquete estadístico STATA 8.0. De las 168 mediciones de tibia y talla, la longitud promedio de la talla es $170 \mathrm{~cm}$ y de tibia $38,42 \mathrm{~cm}$; el coeficiente de correlación de Pearson es 0,88. Al aplicar el modelo de regresión lineal se obtiene la ecuación $\mathrm{T}=66,42+(2.72 \times \mathrm{LMT})$, donde la Talla $(\mathrm{T})$ en cm es igual a $66,42 \mathrm{~cm}$ más el producto de 2,72 por la longitud máxima de la tibia en cm (LMT). Se genera una ecuación en población Colombiana que permite predecir la talla de un individuo desaparecido a partir de la longitud de los restos óseos de su tibia, de esta manera se contribuye al proceso de identificación de sujetos desaparecidos en Colombia.

PALABRAS CLAVE: Tibia; Talla; Mediciones; Violencia.

\section{INTRODUCCIÓN}

En Colombia, la violencia derivada del severo desequilibrio social puede catalogarse como uno de los problemas más graves y estigmatizantes del país, esta violencia generó algo más de 37.000 defunciones en el año 1997, de las cuales 20.000 correspondieron a muertes violentas por homicidio, y aunque la tasa nacional de muertes por este delito ha disminuido de manera progresiva en los últimos años, ya que en el 2004 se alcanzó una tasa de 42 casos por 100.000 habitantes, 8 puntos por debajo a la tasa registrada en el 2003, y casi 30 puntos menos que en el año de 1995 (72.2 x 100.000 hb), todavía se le considera uno de los países mas violentos del mundo, ya que tales cifras sobrepasan ampliamente las de Norte America (19 x 100.000) y Europa (8 x 100.000) siendo éstas solo superadas por Sudáfrica con una tasa de $51 \mathrm{x}$ 100.000 hb (Rodriguez, 2008).

Este gran número de muertes se debe entre otras cosas al aumento progresivo de la delincuencia común, de diversas formas de delincuencia organizada y además a que nuestro país cuenta con un conflicto armado protagonizado por ejércitos irregulares de guerrilla y de paramilitares, que generan masacres, desaparición forzada y las tasas más altas de secuestro del planeta, situaciones que dan origen a una elevada cifra de desaparecidos (7.800 entre los años 1977 y 2004, según Asfaddes, asociación de familiares de desaparecidos) (ASFADDES, 2008).

*MD Patólogo. Antropólogo forense. Director Departamento de Patología, Escuela de Medicina, Facultad de Salud, Universidad Industrial de Santander (UIS). Hospital Universitario de Santander (HUS). Bucaramanga, Colombia.

**Estudiante Interno de Medicina (Estudiante de $6^{\circ}$ año en Medicina), Escuela de Medicina, Facultad de Salud, Universidad Industrial de Santander (UIS), Hospital Universitario de Santander (HUS). Bucaramanga, Colombia.

***Médico Ginecólogo-Obstetra, Grupo de Patología Forense del Instituto Nacional de Medicina Legal y Ciencias Forenses. Regional Nororiente. Bucaramanga, Colombia. 
De los muchos cadáveres que día a día cobra la violencia en Colombia, hay algunos que no logran ser identificados y deben ser inhumados como N. N. en las fosas comunes de los cementerios; otros generalmente víctimas de ejecuciones extrajudiciales, de secuestradores o de paramilitares son abandonados en campos y parajes solitarios donde seguramente nunca van a ser encontrados; estos casos son los que en mayor medida contribuyen a aumentar la cifra de desaparecidos en el país. La desaparición forzada, es definida por la corte penal internacional como la aprehensión, detención o secuestro de una o más personas cometida por un Estado o una organización política, o con su autorización, apoyo o aquiescencia, seguido de la negativa a informar sobre la privación de libertad o dar información sobre la suerte o el paradero de esas personas, con la intención de dejarlas fuera de la ley durante un largo periodo (ONU, 2008).

Con la entrada en vigencia de la Ley de Justicia y Paz (Ley 975 de 2005 de Colombia) como marco jurídico para el proceso de desmovilización de paramilitares en Colombia, un buen número de estos individuos han confesado sus crímenes, lo que ha permitido el descubrimiento de gran cantidad de fosas comunes en todo el territorio nacional y la recuperación de varios cientos de cadáveres, la mayoría de los cuales se encuentran en estado de putrefacción avanzada, mutilados o descuartizados, o en estado de reducción esquelética, situación que hace imposible su identificación fehaciente por el método tradicional del cotejo dactiloscópico o la identificación indiciaria guiada por el reconocimiento de sus facciones, sus señales particulares o sus prendas. La fase inicial de identificación de estos cuerpos, consiste en establecer la cuarteta básica, que busca determinar el sexo, la edad, la talla y el patrón racial del sujeto. El cráneo y la pelvis permiten en la casi totalidad de los casos establecer la diferencias entre individuos masculinos y femeninos. La determinación del patrón racial resulta un tanto complicado en nuestro medio, debido a la casi inexistencia de grupos étnicos puros, sin embargo, el estudio detallado del cráneo ofrece información valiosa en relación con los rasgos raciales predominantes. El estudio de la terminación esternal, de la cuarta costilla, de la sínfisis púbica y de la superficie auricular de ilion, brindan información para establecer de manera aproximada la edad del sujeto al momento de la muerte.

La talla se establece mediante la medición de los huesos largos y la aplicación de formulas diseñadas por diversos investigadores, a partir de poblaciones caucásicas, mongoloides y negroides de otras regiones del mundo. El presente estudio busca determinar si existe correlación entre la longitud de la tibia y la talla en sujetos mestizos colombianos, con el fin de diseñar una ecuación que permita predecir la estatura de un individuo de manera confiable a partir de la longitud de los restos óseos de su tibia.

\section{MATERIAL Y MÉTODO}

Se evaluaron 168 cadáveres de género masculino con edades comprendidas entre los 18 y 50 años, a los cuales se practica autopsia medicolegal en la morgue del Instituto Nacional de Medicina Legal y Ciencias Forenses (INMLYCF) de Bucaramanga, Colombia. Solo se incluyeron sujetos quienes no presentaran deformidad física, lesiones traumáticas o amputación a nivel de los miembros inferiores. A los cadáveres se mide su talla en centímetros y la longitud máxima de la tibia (LMT). La talla del individuo se define como la distancia existente entre el vértice del cráneo y el talón para lo cual se utiliza un tallímetro, posteriormente se realiza una incisión longitudinal desde la región anterior de la rodilla hasta la región media del pie complementada con una incisión transversal sobre la rodilla la cual permite la exposición y disección del extremo superior de la tibia, con la liberación de las diferentes estructuras ligamentosas que la unen al fémur y fíbula, se libera la tibia de los músculos que la rodean y se corta la membrana interósea; finalmente se libera el extremo distal de la tibia de los diferentes ligamentos que la mantienen unida a estructuras vecinas, el hueso así liberado se retira del cuerpo se limpia el exceso de tejidos blandos en ambos extremos y una vez limpio se procede a la medición de la LMT la cual se define como la medida entre el borde del maléolo medial y la parte más prominente del cóndilo lateral de la tibia para lo cual se utiliza una tabla osteométrica que consta de una tabla horizontal calibrada en centímetros $(\mathrm{cm})$, la cual en uno de sus extremos posee una tabla perpendicular de $8 \mathrm{~cm}$ de altura la cual se encuentra fija y en su otro extremo posee una tabla que se desliza sobre su base (Fig.1), luego de la medición de la tibia del sujeto el resto óseo se devuelve a la pierna original sin alterar la morfología del cadáver.

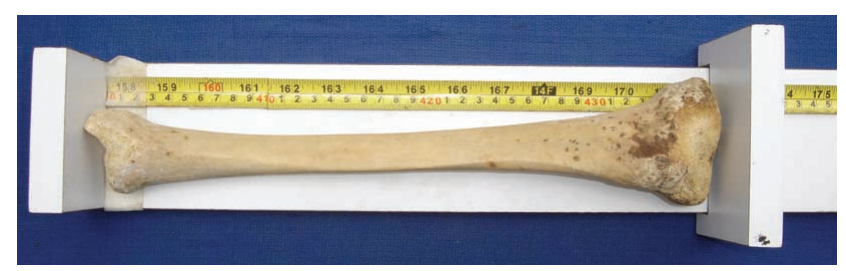

Fig. 1. Imagen de la tabla osteométrica (osteómetro de Broca), utilizado en la estimación de la talla a partir de la medida de la tibia en población Colombiana.

La medición de la talla y la LMT operativamente se lleva a cabo por dos observadores independientes debidamente entrenados previamente para tal tarea, quienes reportan sus medidas en centímetros, cuando existen diferencias en las medidas de cualquiera de las variables entre los observadores mayores a $1,5 \mathrm{~cm}$ se eliminan estos datos del análisis. Se obtienen 168 mediciones de la talla de indivi- 
duos con su respectiva LMT con lo cual se genera una base de datos que permite establecer la correlación existente entre las variables talla y longitud máxima de tibia utilizando el coeficiente de correlación de Pearson, posteriormente para la generación de la ecuación se utiliza el método de regresión lineal $(\mathrm{y}=\mathrm{a}+\mathrm{bx})$ en el cual se practica una regresión de la estatura sobre la talla con el fin de obtener una ecuación que relacione estas dos variables, para cada variable se calculo su respectivo error estándar (EE) con intervalos de confianza al $95 \%$ (IC $95 \%$ ), en todos los análisis se utiliza el programa estadístico STATA 8.0 (Data Analisys and Statistical Software, version 8.0, Stata Corp LP).

\section{RESULTADOS}

Se obtuvieron 168 mediciones de tibia y talla, la longitud promedio en centímetros de la talla es 170,76 con una desviación estándar (DE) de 7,39 cm, la longitud máxima de la tibia (LMT) en promedio es de $38,42 \mathrm{~cm}$ con DE de $2,38 \mathrm{~cm}$ (Tabla I).

Al graficar las variables talla y LMT se obtiene una distribución normal (Fig. 2) y se obtiene un coeficiente de correlación de Pearson de 0,88 .
$\mathrm{Al}$ aplicar el modelo de regresión lineal $(\mathrm{y}=\mathrm{a}+\mathrm{bx})$ se obtiene la siguiente ecuación:

$$
\begin{gathered}
T=66,42+(2.72 * L M T) \\
(4,5) \quad 0,12)
\end{gathered}
$$

Donde la Talla (T) en cm es igual a 66,42 cm más el producto de 2,72 por la longitud máxima de la tibia (LMT) en $\mathrm{cm}$, entre paréntesis se describen los respectivos errores estándar (EE) en cm.

\section{DISCUSION}

La estatura se considera exclusiva de la especie humana por cuanto los otros animales no asumen una postura erguida habitual fisiológica, sus dimensiones dependen de varios segmentos como el cefálico, raquídeo (altura de la columna), pelviano y de los miembros inferiores, cada uno contribuye a la talla del individuo en consideración a la edad, sexo, raza, condiciones socioeconómicas y sicosociales, y finalmente las tendencias históricas (Rodriguez, 1994).

Dentro del proceso histórico se han desarrollado diversos métodos matemáticos en diferentes lugares del mun-

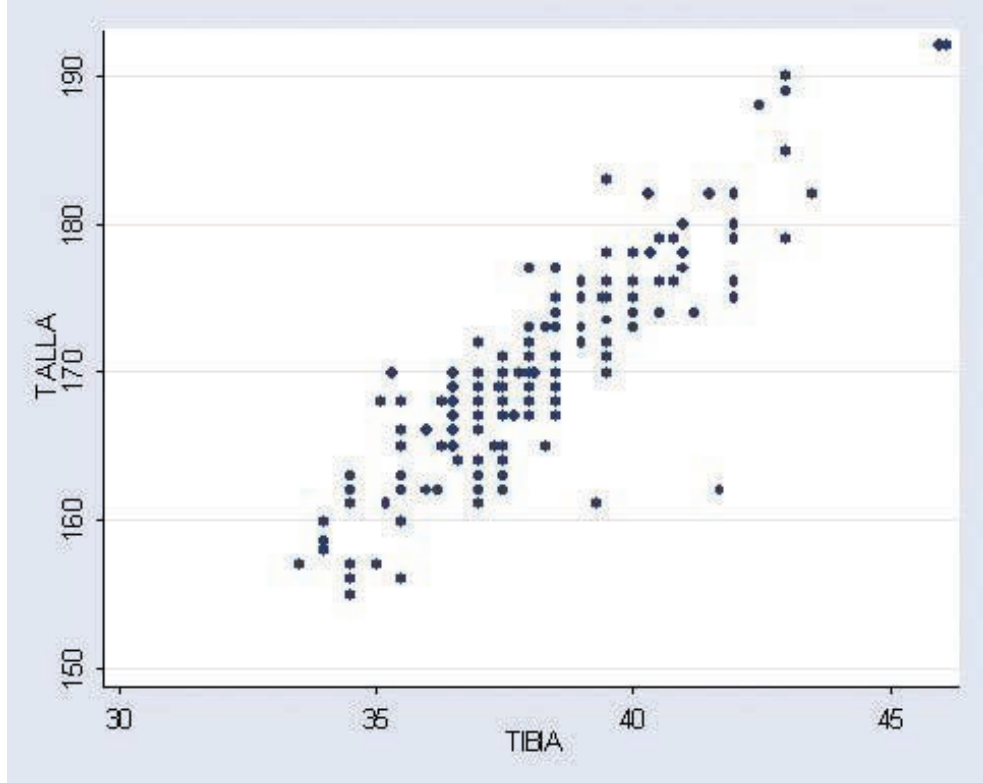

Fig. 2. Distribución normal de tibia y talla en 168 cadáveres de género masculino con edades entre los 18 y 50 años autopsiados en la morgue del Instituto Nacional de Medicina Legal y Ciencias Forenses (INMLYCF) de Bucaramanga, Colombia.

Tabla I. Mediciones de tibia y talla en 168 cadáveres de género masculino con edades entre los 18 y 50 años autopsiados en la morgue del Instituto Nacional de Medicina Legal y Ciencias Forenses (INMLYCF) de Bucaramanga, Colombia.

\begin{tabular}{lccccc}
\hline VARIABLE & DATOS $(\mathbf{n})$ & MEDIA $(\mathbf{X})$ & DE & MÍNIMA & MÁXIMA \\
\hline Talla $(\mathrm{cm})$ & 168 & 170,76 & 7,39 & 155 & 192 \\
Tibia $(\mathrm{cm})$ & 168 & 38,42 & 2,37 & 33,5 & 46,1 \\
\hline
\end{tabular}


do con el fin de predecir la talla a partir de la longitud de restos óseos. Así Fully (1956) determinó la estatura sumando los segmentos óseos que contribuyen a la longitud vertical del esqueleto (Altura del cráneo, altura de los cuerpos vertebrales, longitud del fémur, tibia y altura de calcáneo y astrágalo articulados) y realizando una corrección por los tejidos blandos correspondientes, entre los cuales se media la longitud de la tibia excluyendo las eminencias intercondilares. Posteriormente hacia el año de 1960 junto con Pineau (Fully \& Pineau, 1960) desarrollan una técnica donde obtenía la estatura a partir de la suma del segmento vertebral lumbar y la tibia o el fémur sin necesidad de correcciones por tejido blando, en ambos métodos se utilizó la misma población que consistió en 164 esqueletos masculinos Europeos provenientes de campos de concentración durante la segunda guerra mundial. Feldesman \& Fountain (1996) concluyen que la proporción fémur y estatura son diferentes en los negros con respecto a blancos y asiáticos, sin embargo en la practica el uso de estas formulas para estimar la talla es limitado. Un estudio llevado en Guatemala concluye que existen diferencias estadísticamente significativas en la estimación de la talla al utilizar cada uno de los métodos y recomienda que las formulas generadas por esos métodos no deben emplearse en el análisis forense de esqueletos en Guatemala (Ríos, 2003). Además Troter \& Gleser (1977) proponen que en periodos cortos de tiempo hay cambios significativos en las dimensiones de segmentos corporales dentro de una misma población por lo cual se hace necesario generar ecuaciones para predecir la talla en cada población, por los hechos anteriores surge la necesidad que se establezcan en las diferentes poblaciones métodos adecuados con respecto a la longitud de diferentes segmentos óseos para predecir la estatura.

Las dimensiones de los miembros inferiores han sido objeto de análisis en tales estudios y se ha concluido que pueden ser usadas para calcular la estatura de una manera confiable (Ozaslan et al., 2003). Según Lundy los huesos largos mas exactos de la extremidad inferior para determinar la estatura son la tibia y el fémur (Lundy, 1985), con coeficientes de correlación de 0,94 y 0,95 respectivamente (Radoinova et al., 2002; Hauser et al., 2005), aunque en el estudio realizado el coeficiente de correlación es de 0,88 lo consideramos apropiado, nuestra apreciación es que la tibia es un hueso largo que en diversos estudios como en el nuestro ha mostrado correlaciones satisfactorias para estimar la talla.

Con respecto al error estándar (EE) manejado se debe tener en cuenta que después de la muerte la estatura disminuye para los hombres en $1,2 \mathrm{~cm}$ y en el caso de las mujeres $2 \mathrm{~cm}$, los manejados en nuestra investigación son aceptables.

Se concluye que la longitud máxima de la tibia muestra una excelente correlación con la estatura lo cual permite que sea usada para generar ecuaciones que permitan estimar la estatura no solo en el contexto de los desaparecidos sino en otras áreas de ciencias de la salud además se deben llevar a cabo nuevos procesos investigativos con otros segmentos óseos y en diversas poblaciones latinoamericanas.

MANTILLA, H. J. C.; CÁRDENAS, D. N. \& JÁCOME, B. J. M. Estimation of height from measurements of the tibia in Colombian population. Int. J. Morphol., 27(2):305-309, 2009.

SUMMARY: Enforced disappearance is a common phenomenon in Colombia, appearing daily human remains in mass graves, the state has the responsibility to identify those remains, the initial process of identification is required to determine gender, race, age and height. Our goal is to establish the correlation between the length of the tibia and the height of an individual in order to generate an equation to predict the size of an individual from his tibia skeletal remains. We evaluated 168 corpses who are medico-legal autopsy practice, aged between 18 and 50 years olds. It makes measuring the height of the subject and the length of the tibia after dissection, it is estimated the correlation between these two variables from the Pearson correlation and applies a linear regression model to generate an equation to predict the size of an individual from the length of the tibia using the statistical package STATA 8.0. Were obtained 168 tibia and height measurements, the average length of the height is $170 \mathrm{~cm}$ and tibia $38.42 \mathrm{~cm}$, Pearson correlation coefficient is 0.88 . By applying the linear regression model is obtained by the equation $\mathrm{H}=66.42+(2.72 \mathrm{x}$ MLT), where height $(\mathrm{H}) \mathrm{cm}$ is equal to $66.42 \mathrm{~cm}$ plus the product of 2.72 by the maximum length of tibia in $\mathrm{cm}$ (MLT). We generate an equation that predicts Colombian population height of an individual missing from the length of his tibia, thus contributing to the process of identification of missing in Colombia.

KEY WORDS: Tibia; Height; Violence; Body weights and measures.

\section{REFERENCIAS BIBLIOGRÁFICAS}

ASFADDES, Asociación de familiares de detenidos desaparecidos. Disponible en http://www.asfaddes.org.co, 2008.
Feldesman, M. R. \& Fountain, R. L."Race" specificity and the femur/stature ratio. Am. J. Phys. Anthropol., 100(2):207-24, 1996. 
MANTILLA, H. J. C.; CÁRDENAS, D. N. \& JÁCOME, B. J. M. Estimación de la talla a partir de la medida de la tibia en población Colombiana. Int. J. Morphol., 27(2):305-309, 2009.

Fully, G. New method of determination of the height. Ann. Med. Leg. Criminol. Police. Sci. Toxicol., 36(5):266-73, 1956.

Fully, G. \& Pineau, H. Determination of the height by means of the skeleton. Ann. Med. Leg. Criminol. Police. Sci. Toxicol., 40:145-53, 1960.

Hauser, R.; Smolinski, J. \& Gos, T. The estimation of stature on the basis of measurements of the femur. Forensic Sci. Int., 147(2-3):185-90, 2005.

Lundy, J. K. The mathematical versus anatomical methods of stature estimate from long bones. Am. J. Forensic Med. Pathol., 6(1):73-6, 1985.

ONU, Organización de las Naciones Unidas. Disponible en http://www.uno.org, 2008.

Ozaslan, A.; Iscan, M. Y.; Ozaslan, I.; Tugcu, H. \& Koc, S. Estimation of stature from body parts. Forensic Sci. Int., 132(1):40-5, 2003.

Radoinova, D., Tenekedjiev, K. \& Yordanov, Y. Stature estimation from long bone lengths in Bulgarians. Homo., 52(3):221-32, 2002.

Ríos, L. Estimación de la estatura en restos óseos: Problemas metodológicos. En: XVII Simposio de Investigaciones Arqueológicas en Guatemala, 2003. Laporte, J. ed. Guatemala, Museo Nacional de Arqueología y Etnología, 2003. pp.110-16.

Rodriguez, J. V. Reconstrucción de la estatura. En: Introducción a la Antropología Forense: Analisis e identificacion de restos oseos humanos. Bogotá, Universidad Nacional de Colombia Santafé de Bogotá, 1994.

Rodriguez, M. A. Variación estacional de la mortalidad por homicidio en Colombia, 1985 a 2001. Colomb. Med. 39:154-60, 2008.

Trotter, M. \& Gleser, G. C. Corrigenda to: estimation of stature from long limb bones of American Whites and Negroes, American Journal Physical Anthropology (1952). Am. J. Phys. Anthropol., 47(2):355-6, 1977.
Dirección para correspondencia:

Dr. Nelson Cárdenas Duran.

Carrera 48 No 28-65

Bucaramanga

COLOMBIA

Telefono: 57-7-6326846

E-mail: ncardenas@hotmail.com

Recibido : 10-09-2008

Aceptado: 22-01-2009 
